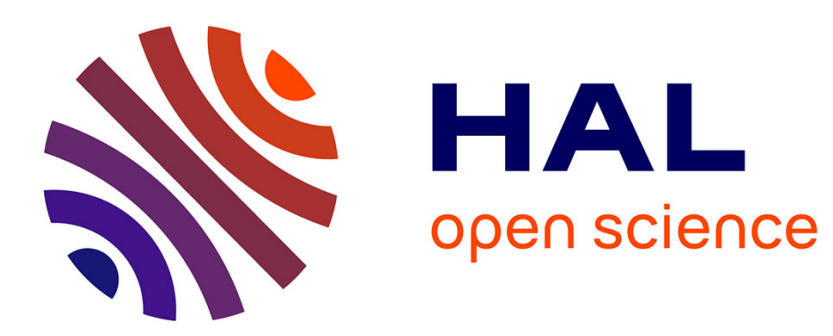

\title{
Using the moving synchronous average to analyze fuzzy cyclostationary signals
}

Q. Leclere, Nacer Hamzaoui

\section{To cite this version:}

Q. Leclere, Nacer Hamzaoui. Using the moving synchronous average to analyze fuzzy cyclostationary signals. Mechanical Systems and Signal Processing, 2014, 44, pp.149-159. 10.1016/j.ymssp.2013.01.005 . hal-00915261

\section{HAL Id: hal-00915261 \\ https://hal.science/hal-00915261}

Submitted on 29 Mar 2016

HAL is a multi-disciplinary open access archive for the deposit and dissemination of scientific research documents, whether they are published or not. The documents may come from teaching and research institutions in France or abroad, or from public or private research centers.
L'archive ouverte pluridisciplinaire HAL, est destinée au dépôt et à la diffusion de documents scientifiques de niveau recherche, publiés ou non, émanant des établissements d'enseignement et de recherche français ou étrangers, des laboratoires publics ou privés. 


\title{
Using the moving synchronous average to analyse fuzzy cyclostationary signals
}

\author{
Quentin Leclère ${ }^{\mathrm{a}, *}$ Nacer Hamzaoui ${ }^{\mathrm{a}}$ \\ ${ }^{a}$ Laboratoire Vibrations Acoustique, INSA Lyon, 25 bis avenue Jean Capelle \\ F-69621 Villeurbanne Cedex, FRANCE
}

\begin{abstract}
Cyclostationarity is a property of vibration and acoustic signals recorded on rotating machines operating at constant speed. It states that the statistic properties of signals are periodic: the random process defined by the signal observed at a given position in the cycle is stationary, the cycle being defined as the angle interval between two identical configurations of the mechanical system. Cyclostationarity is not fully satisfied if the signals are acquired in the time domain on rotating machines with a fluctuating rotation speed. Indeed, if the instantaneous rotation speed is not purely periodic, it means that time samples taken at a constant time interval (equal to the average cycle duration) do not correspond exactly to an angle in the cycle. In this particular case, a synchronous averaging of cycle realizations can still be processed to estimate a periodic part using a predefined trigger angle to align cycle realizations before the averaging process. In these conditions, the synchronous average depends on the chosen synchronization angle : each point of the synchronous average is an estimate of the expected value of the signal at a given time preceding or following the synchronization angle. The synchronous average can be computed as a function of the synchronization angle, varying over an entire cycle. The result is a moving synchronous average that can be post-processed for diagnosis purposes. For example, a time frequency representation of the moving synchronous average can be computed, and the synchronization angle maximizing each point of the time frequency map can be easily extracted. Under certain conditions of instantaneous speed fluctuations, this analysis allows the precise localization of different mechanical events in the cycle, as well as their contributions in the analyzed vibration or acoustic signal. The analysis of the moving synchronous average leads also to the estimation of the energy loss of the synchronous average processed in angle caused by cycle to cycle speed fluctuations.
\end{abstract}

Key words: Fuzzy cyclostationarity, synchronous average, angular sampling, instantaneous rotation speed, diesel engine diagnosis, diesel engine noise

$\bar{*}$ Corresponding author. Fax: 33.4.72.43.87.12. E-mail address: 


\section{Nomenclature}

\begin{tabular}{|c|c|}
\hline$t$ & time history \\
\hline$\theta$ & (unwrapped) rotation angle \\
\hline$\Theta$ & angular cycle length \\
\hline$\widehat{\Omega}$ & average rotation speed \\
\hline$\Omega(\theta)$ & Instantaneous Angular Speed (IAS) as a function of $\theta$ \\
\hline$f(\theta)$ & elapsed time as a function of $\theta$ \\
\hline $\bar{\Omega}(\theta)$ & IAS of the mean cycle $(\theta \in[0 \ldots \Theta[)$ \\
\hline$\sigma_{\nu}(\theta)$ & normalized cycle to cycle fluctuation of the IAS $(\theta \in[0 \ldots \Theta[)$ \\
\hline $\bar{f}(\theta)$ & elapsed time as a function of $\theta$ for the mean cycle $(\theta \in[0 \ldots \Theta[)$ \\
\hline $\bar{T}$ & duration of the mean cycle \\
\hline $\operatorname{ADP}(\theta)$ & Angular Deterministic Part $\theta \in[0 \ldots \Theta[$ \\
\hline $\mathrm{TDP}_{\theta}(t)$ & $\begin{array}{l}\text { Temporal Deterministic Part }(t \in[-\infty \ldots \infty[) \text { for the } \\
\text { synchronization angle } \theta \in[0 \ldots \Theta[\end{array}$ \\
\hline $\operatorname{ASA}(\theta)$ & Angular Synchronous Average $(\theta \in[0 \ldots \Theta[)$ \\
\hline $\mathrm{TSA}_{\theta}(t)$ & $\begin{array}{c}\text { Temporal Synchronous Average, } t \in[-\bar{T} / 2 \ldots \bar{T} / 2[ \\
\text { for the synchronization angle } \theta \in[0 \ldots \Theta[\end{array}$ \\
\hline $\operatorname{MTSA}(\theta, t)$ & Moving TSA, $\theta \in[0 \ldots \Theta[, t \in[-\bar{T} / 2 \ldots \bar{T} / 2[$ \\
\hline$s_{r m s}(t)$ & RMS value over a short sliding time window of $s(t)$ \\
\hline$s_{t f m}(t, f)$ & time frequency distribution of $s(t)$ \\
\hline
\end{tabular}

\section{Introduction}

The synchronous average is a signal processing tool consisting in averaging several portions of signal chosen in function of the studied mechanical system[1]. For cyclostationary signals, the synchronous average is an estimation of the deterministic or periodic part [2]. It can also be regarded through comb filtering [3], the signal is filtered by a specific comb resulting in an averaging process over signal samples separated by a specific delay. Noise and vibration signals recorded on rotating machines are determined by cyclic excitations quentin.leclere@insa-lyon.fr. 
defined in angle, and the response of the structure is defined by its time domain impulse response, the determinism of such signals being consequently dual [4]. If the studied rotating machine is operating with a strictly constant or purely periodic IAS (Instantaneous Angular Speed), the time and angular clocks are totally dependent, and this duality is masked ; processing signals in time or angle will lead to equivalent results. If not, the duality leads to so-called fuzzy-cyclostationary signals [5], and the result of synchronous averaging will be different in time or angle. The fuzzy-cyclostationary concept is established in the first section of this paper. The time-domain synchronous average is redefined in this context in the second part of this work. It requires especially to define a synchronization angle, and its computation for several synchronization angles in the cycle is defined here as the moving synchronous average. The concepts proposed in this study are illustrated by two experimental examples presented in the third section. The fourth part shows how ASA and TSA (Angular and Temporal Synchronous Averages) can be compared. Then, the moving synchronous average is introduced, and different post processing tools are proposed to extract some information about the studied rotating machine.

\section{A definition of Fuzzy Cyclostationarity}

The concept of Fuzzy Cyclostationarity is deeply inspected in reference [5] (in French). A brief definition is however given here, in order to clearly establish the general framework of this study.

Let $\omega(t)$ represent the instantaneous angular speed (IAS, $\mathrm{rad} / \mathrm{s}$ ) of a rotating machine as a function of time, and $f$ the bijection between the temporal and angular domains such as $t=f(\theta)$. The instantaneous angular speed can be expressed as a function of the angle :

$$
\Omega(\theta)=\omega(f(\theta))
$$

In stationary operating conditions, $\Omega(\theta)$ is assumed to be cyclostationary of cycle length $\Theta$. The bijection $f$ can be expressed as a function of $\Omega$ :

$$
f(\theta)=t=\int_{0}^{t} d \tau=\int_{0}^{\theta} \frac{d \alpha}{\Omega(\alpha)}
$$

The statistical properties of $\Omega(\theta)$ are $\Theta$-periodic. The angle to time relationship $f(\theta)$ can be written at $\theta+\Theta$ as follows :

$$
f(\theta+\Theta)=\int_{0}^{\theta} \frac{d \alpha}{\Omega(\alpha)}+\int_{\theta}^{\theta+\Theta} \frac{d \alpha}{\Omega(\alpha)}=t+T(\theta)
$$


The IAS at $\theta+\Theta$ is thus equal to

$$
\Omega(\theta+\Theta)=\omega(f(\theta+\Theta))=\omega(t+T(\theta))
$$

If $T(\theta)=T$ is constant, it means that the cyclostationarity of $\Omega$ in angle implies the cyclostationarity of $\omega$ in time. According to Eq. (3), this is the case if $\Omega(\theta)$ is constant or periodic. If the cyclic variance of $\Omega(\theta)$ is not null, then $T(\theta)$ depends on $\theta$ and some fluctuations of the cycle length will appear in time. In this case, $\omega(t)$ keeps the property of cyclostationarity, but with a cycle length equal to the average value of $T(\theta)$. It means that cycle realizations in the time domain will not correspond exactly to a cycle length $\Theta$ in angle : that's why this kind of cyclostationary process is qualified as fuzzy.

An important consequence of fuzzy cyclostationarity is that cyclic estimators, like the cyclic average or variance, will lead to different results if processed using a constant time or angle sampling. The present work aims to study these differences, and to present how the fuzzy cyclostationarity property can be used for diagnosis purposes.

\section{The synchronous average : an estimation of the deterministic part of the signal}

\subsection{Synchronous averaging of cyclostationary signals}

Cyclostationary signals are characterized, by definition, by periodical statistic properties. For signals recorded on mechanisms, this periodicity is a mechanical periodicity, i.e. the duration between two identical geometrical configurations of moving parts of a rotating machine (that will be designed in the following by 'cycle'). If the machine is operating with a constant (or purely periodic) rotation speed, the cycle length can be defined in time or angle, and signals can be processed either with a time or an angular sampling. The synchronous average (also called cyclic average) is defined as the average value of the signal at a given position in the cycle. It is in fact an estimation of the expected value of the signal, assessed over an entire cycle. The relation with the determinism is then straightforward : the deterministic part corresponds to what can be expected at a given time from the knowledge of what happened before, particularly the cycle position reached by the mechanism. This deterministic part is thus naturally defined as the expected value of the signal during a cycle :

$$
d(\theta)=E[s(\theta+k \Theta)], \quad k \in \mathbb{Z},
$$

where $s$ and $d$ represent, respectively, the cyclostationary signal and its deterministic part, and $\Theta$ the cycle length. If the rotation speed of the machine is 
constant or purely periodic, the time-angular correspondence is deterministic : $\theta$ can be replaced by $t$ and $\Theta$ by $\Theta / \widehat{\Omega}$ (with $\widehat{\Omega}$ the average rotation speed).

The definition of the deterministic part of a signal leads to define also the random part (also called the centered signal), difference between the signal and its deterministic part :

$$
r(\theta)=s(\theta)-d(\theta)
$$

This random part is of prime interest, for example, for some source separation problems where removing the deterministic part can decrease high coherences between inputs [6].

\subsection{Synchronous averaging of fuzzy cyclostationary signals}

If the rotation speed of the machine is not constant (or not purely periodic), the bijective relationship between the time and angular clocks is lost in the cycle. The definition of the deterministic part it then different in the time and angle domains. In angle, the deterministic part remains the expected value of the signal at a given position in the cycle (it will be noted ADP for angle deterministic part in the following, and its estimate is the angular synchronous average (ASA)). In the time domain, the deterministic part can be defined as

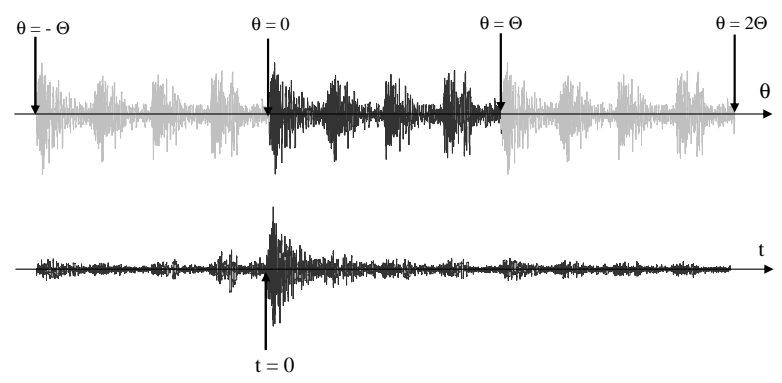

Fig. 1. Top : Angular deterministic part. Bottom : temporal deterministic part, synchronization angle $\alpha=0$.

the expected value of the signal at a given time distance of a synchronization angle $\alpha$ :

$$
d_{\alpha}(t)=E\left[s\left(T_{\alpha+k \Theta}+t\right)\right], \quad k \in \mathbb{Z},
$$

where $\alpha$ is the synchronization angle $(\alpha \in[0, \ldots \Theta])$, and $T_{\alpha+k \Theta}$ the time corresponding to the angle $\alpha+k \Theta$ (angle $\alpha$ of cycle $k$ ). Practically, the trigger dates $T_{\alpha+k \Theta}$ can be estimated using an angle encoder. This definition of the TDP (temporal deterministic part) is thus function of the synchronization angle $\alpha$. Moreover, the TDP is not periodic by construction, it is theoretically defined for $t \in]-\infty ;+\infty[$. An illustration of TDP and ADP is given in figure 1 . 
The interest of this time domain approach relies in the the duality of the determinism of fuzzy cyclostationary signals : the excitation occurrence is mainly governed in angle, while the vibro-acoustic response of the structure is defined in time. The effects of both approaches are compared theoretically and experimentally in [4]. The ADP gives generally the most energetic output on the whole cycle, except in the part of the cycle following the synchronization angle : the TDP captures more efficiently the response of the structure to excitations occurring in the vicinity of the synchronization.

\section{Presentation of experimental cases}

The concepts presented in this work will be illustrated by two different experimental examples. The first one deals with a 4 cylinder diesel engine operating at cold idle (fig. 2, left). The cycle length is 720 deg. CA (Crankshaft Angle), corresponding to the four strokes. The second experiment is a gear test rig with two shafts (fig. 2, right). The first shaft, driven by an electric motor, is mounted with the first gear (45 teeth) and the second shaft with the driven gear (24 teeth). One tooth of the driven gear is broken to generate a typical gear fault. The cycle length is 2880 degrees, or 8 revolutions of the driving shaft. During one cycle, the driving shaft achieves exactly 8 revolutions and the driven shaft exactly 15. Physical parameters of each test rig are provided
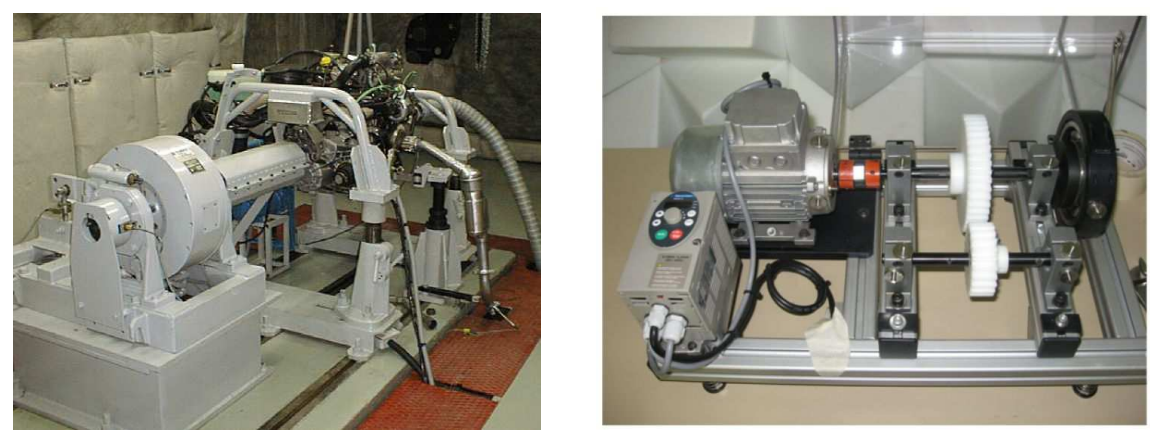

Fig. 2. Test rigs used for signals and results shown in this work. Left : Diesel engine, right : gears.

in table 1. For both rigs, three signals are collected from the angle encoder, one accelerometer and one microphone. In both cases, one record only is considered for either angular or temporal approaches. The signals are recorded using a time sampling, and then resampled in angle using the angle encoder output (cf. [7]). The IAS of the mean cycle, as introduced in appendix, is drawn in figure 3. The cycle-to-cycle fluctuations of the IAS are represented by the \pm 2 standard deviation confidence intervals. For the diesel engine, there is a significant periodic speed variation within a cycle (more than $10 \%$ of the average speed), caused by the 4 combustions occuring each 180 degrees CA. The speed 


\begin{tabular}{|c|c|c|}
\hline Test rig & diesel engine & gears \\
\hline description & $\begin{array}{c}4 \text { cylinders } \\
1.9 \mathrm{~L} \mathrm{dci}\end{array}$ & $\begin{array}{c}\text { driving gear }: Z=45, \\
\text { driven gear } Z=24\end{array}$ \\
\hline angular coder location & crankshaft & driving shaft \\
\hline average rotation speed $(\mathrm{rpm})$ & 850 & 270 \\
\hline Inst. speed fluctuation $\sigma_{\nu}(\%)$ & 0.5 & 2.4 \\
\hline cycle length & 2 rotations & 8 rotations \\
\hline time sampling $(\mathrm{Hz})$ & 102400 & 50000 \\
\hline record length & 30 s $(212$ cycles $)$ & 120 s $(68$ cycles $)$ \\
\hline angular coder resolution $($ degrees $)$ & 0.5 & 0.5 \\
\hline
\end{tabular}

Table 1

Physical \& acquisition parameters for both test rigs
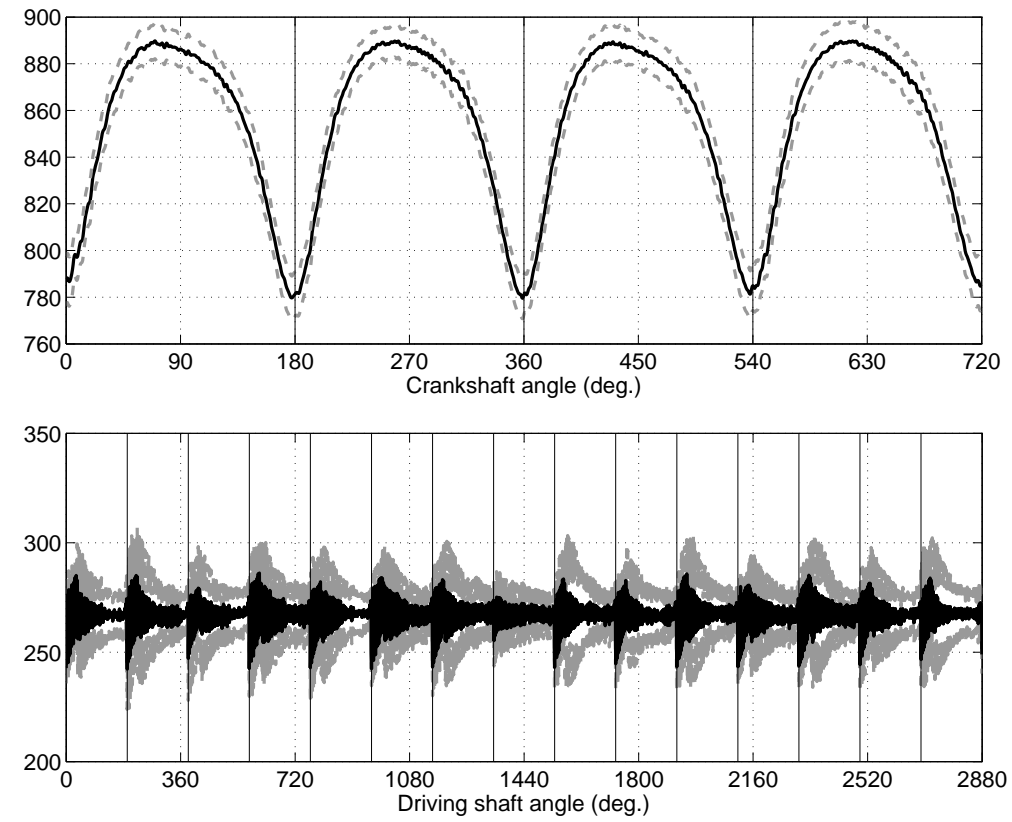

Fig. 3. Averaged instantaneous cycle speed (black) and \pm 2 standard deviation (gray). Top : diesel engine (vertical black lines standing for TDCs), Bottom : gears (vertical black lines standing for the missing tooth crossing the driving gear).

fluctuation level is much lower, with a normalized standard deviation of about $0.5 \%$. Considering the gears test rig, the periodic speed variations (about 15\% of the averaged speed) are mainly caused by the defect itself (missing tooth), occuring 15 times by cycle. This defect acts like a periodic chock on both gears, inducing a vibratory response in angle of the shaft. This response is dominated by a mode at about $480 \mathrm{~Hz}$, responsible of oscilation bursts appearing in the intantaneous cycle speed. The standard deviation of the instantaneous speed 
varies between $1.5 \%$ and $3 \%$ within a cycle.

\section{Comparing angular and temporal deterministic parts}

It is not easy to compare ADP and TDP for several reasons. Firstly, the TDP is not periodic. Secondly, both signals are discrete, the first one being defined with a constant step in angle and the second one in time. A solution, in order to be able to compare ADP and TDP, is to resample the ADP in time, using either one cycle realization of IAS or the IAS of the mean cycle (see the definition in appendix). Then, the TDP can be truncated to adjust its length to same duration as the ADP.

The RMS value of ADP and TDP is drawn in figure 4, for the microphone signals of both experimental cases. These RMS values are computed over a short sliding time window, to keep a good resolution of energy variations within the cycle :

$$
s_{r m s}(t)=\sqrt{\frac{1}{L} \int_{-L / 2}^{L / 2} s(t-\tau)^{2} d \tau},
$$

where $L$ is the length of the time window, adjusted to $2.5 \mathrm{~ms}$ in this work. The synchronization angle is 0 , corresponding to a TDC for the engine test rig, and to an impact due to the missing tooth for the gear test rig. Considering the engine signals, during the stroke following the synchronization, the most energetic signal is the TSA because the main contribution results from the combustion occurring at $\theta=0$. For the three other strokes, the ASA is more energetic than the TSA. Considering the gear rig signals, 15 energy bursts are clearly visible on the ASA, corresponding to the 15 cycle occurrences of the gear fault. The TSA is much more energetic during the angle length following the impact occurring at the synchronization angle, and remains more energetic than the ASA during about 540 degrees.

\section{The moving synchronous average}

6.1 Angular resolution of the moving temporal synchronous average (MTSA)

The TDP is assessed for one particular synchronization angle, using the temporal synchronous average (TSA). It can thus be interesting to look at it in function of this synchronization angle : this result will be designated by the moving temporal synchronous average (MTSA). An important point concerns the resolution of this MTSA, in terms of the synchronization angle. The bias of the TDP for an event occurring at an angle $\theta$ of the synchronization is [4] : 

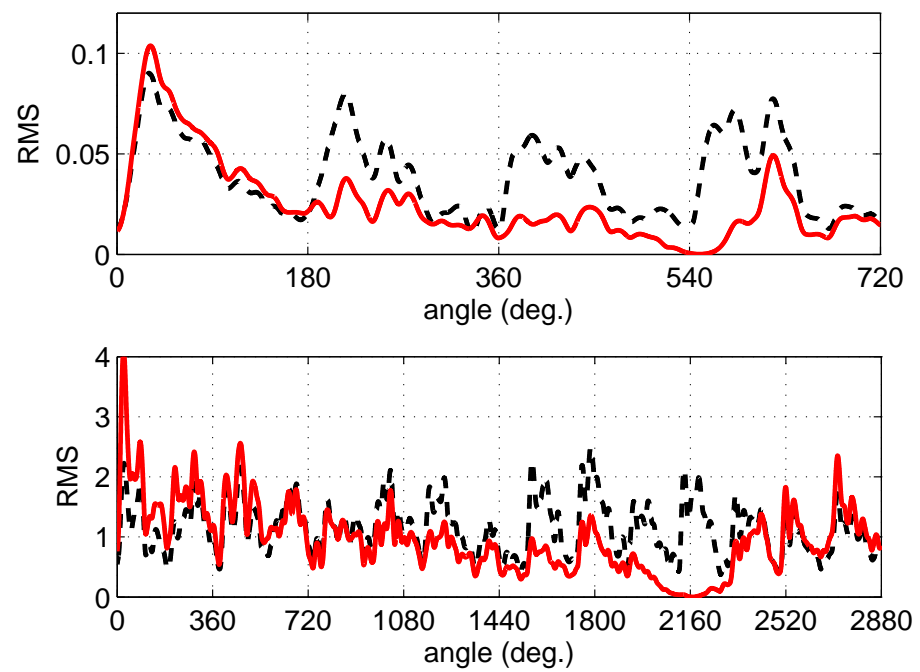

Fig. 4. Comparison between the RMS value of the ASA(dashed black) and TSA (solid red, $\alpha=0$ ) of the microphone signal. Top : Diesel engine, Bottom : gears.

$$
B\left(f, \theta, \sigma_{\nu}, \widehat{\Omega}\right)=e^{-2 \pi^{2} f^{2} \theta^{2}\left(\sigma_{\nu}\right)^{2} / \widehat{\Omega}^{2}}
$$

Where $\widehat{\Omega}$ and $\sigma_{\nu}$ stand for, respectively, the average angular speed and the normalized standard deviation of the instantaneous speed. A threshold can be adjusted to this bias factor to set the synchronization angle resolution :

$$
B\left(f, \theta, \sigma_{\nu}, \widehat{\Omega}\right)>0.95
$$

that leads to

$$
\Delta \theta<\frac{\widehat{\Omega}}{\pi \sigma_{\nu} f_{\max }} \sqrt{\frac{\log (0.95)}{-2}},
$$

where $f_{\max }$ stands for the upper bound of the frequency band of interest. Practically, it means that the difference between two TSA separated by less than $\Delta \theta$ is less than $0.5 d B$.

\subsection{Time-frequency maximization of the MTSA}

The TSA of the response to a transient excitation is theoretically maximum when the synchronization angle coincides with its angle occurrence. The difficulty is that several events exist within a cycle, the TSA is thus maximum after the synchronization angle and before the occurrence of the following event. The search of the synchronization angle maximizing the TSA can thus provide information about the angle occurrence of the event dominating the signal. 


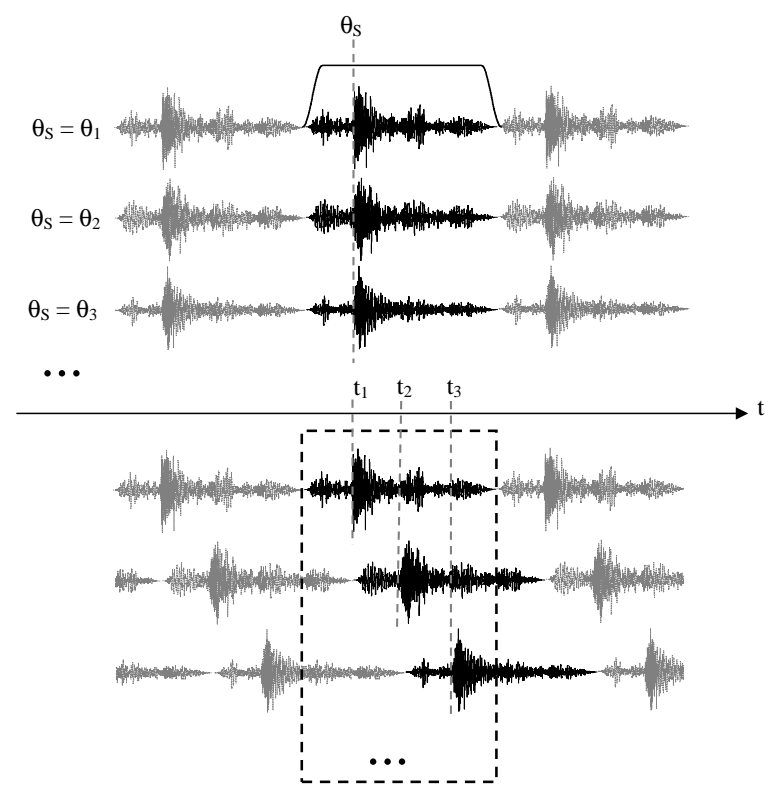

Fig. 5. Computation of the MTSA. top : windowed and periodized TSA computed for different synchronization angles. bottom : synchronized TSAs, and selection of synchronized TSAs during one cycle (materialized by the dashed black box).

This work requires the synchronization of the TSAs processed for each synchronization angle : the $t=0$ of each TSA corresponding to the occurrence of its synchronization angle. This operation is realized using the IAS of the mean cycle (cf. appendix), that leads to analyze a typical (average) cycle realization. It provides a time to angle correspondence $\bar{f}(\theta)$, allowing to time-shift different TSA in function of their synchronization angle. Moreover, TSA have to be truncated, their total duration have to be adjusted to the length of the averaged duration of a cycle, noted $\bar{T}$. TSA can then be periodized, so as to be compared over an entire cycle. A windowing can be used (Tukey for example) to avoid discontinuities generated by the periodization. Basic principles to compute the moving synchronous average are given in figure 5 and a flowchart of the main computation steps are given in figure 6 .

Once TSA at different synchronization angles are computed and synchronized, it can be interesting to search, during the cycle, the sync. angles maximizing the RMS value of the MTSA :

$$
\theta_{r m s}^{\max }(t)=\underset{\theta \in[0 \ldots \Theta[}{\operatorname{argmax}}\left(\operatorname{MTSA}_{r m s}(\theta, t)\right),
$$

where $\operatorname{MTSA}_{r m s}(\theta, t)$ is the short-time RMS value of the MTSA at the synchronization angle $\theta$ as expressed in equation 8. The maximized MTSA RMS value is thus equal to

$$
\operatorname{MTSA}_{r m s}^{\max }(t)=\operatorname{MTSA}_{r m s}\left(\theta_{r m s}^{\max }(t), t\right)
$$




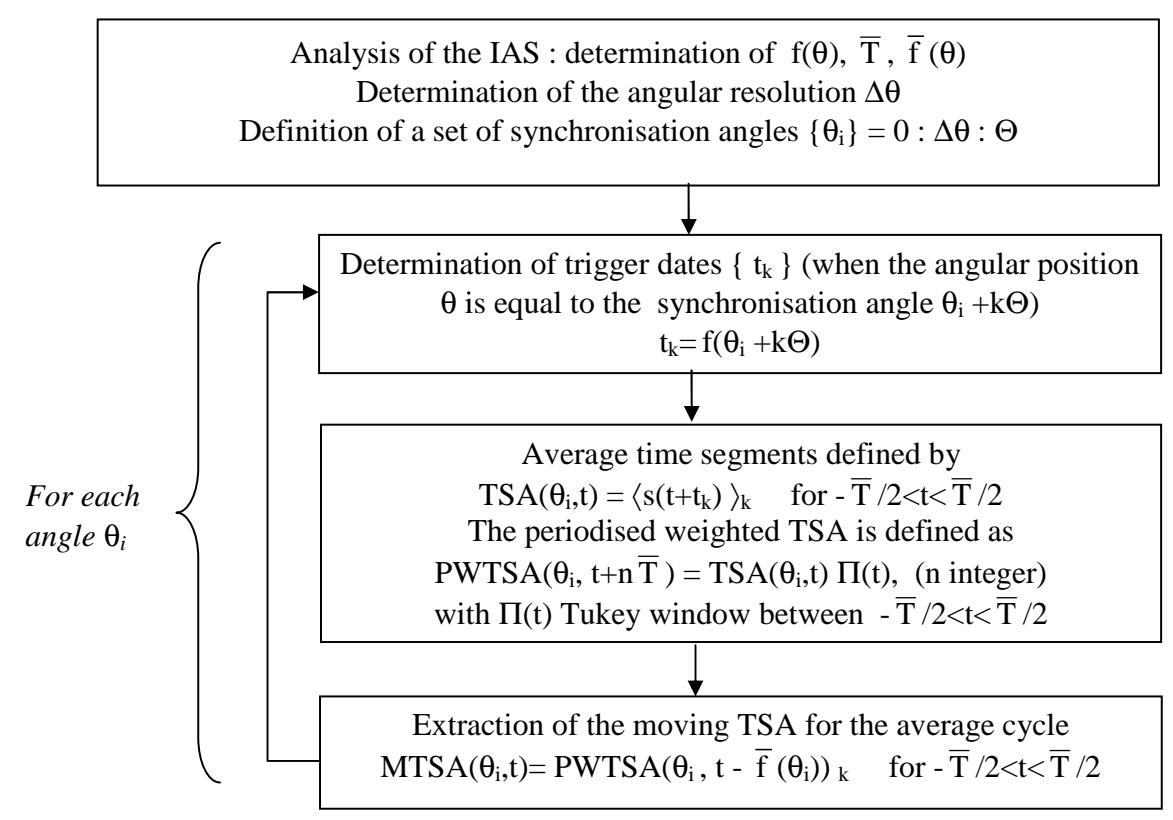

Fig. 6. Main computation steps for the estimation of the MTSA.

This operation is realized, in figures 7 and 8 (top), for the microphone signal in both experimental cases. The maximized RMS value is compared to the RMS value of the ASA, for the same signal. The synchronization angle
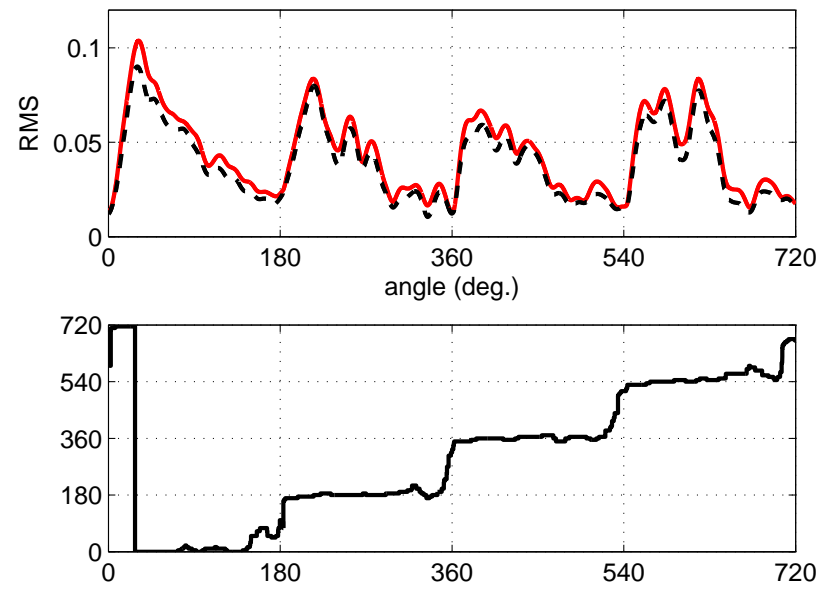

Fig. 7. Diesel engine test rig. Top : RMS value of the ASA(dashed black) and maximized MTSA (solid red) of the microphone signal. Bottom : corresponding synchronization angle maximizing the MTSA.

maximizing the MTSA is also drawn in figures 7 and 8 (bottom). Some steps are observed for both experimental cases, indicating the angle occurrence of the event dominating the signal. 4 steps are visible in figure 7 , indicating the occurrence of the 4 combustions of the engine cycle. 15 steps are observed in figure 8 , indicating the 15 chocks generated by the missing tooth on the driven 

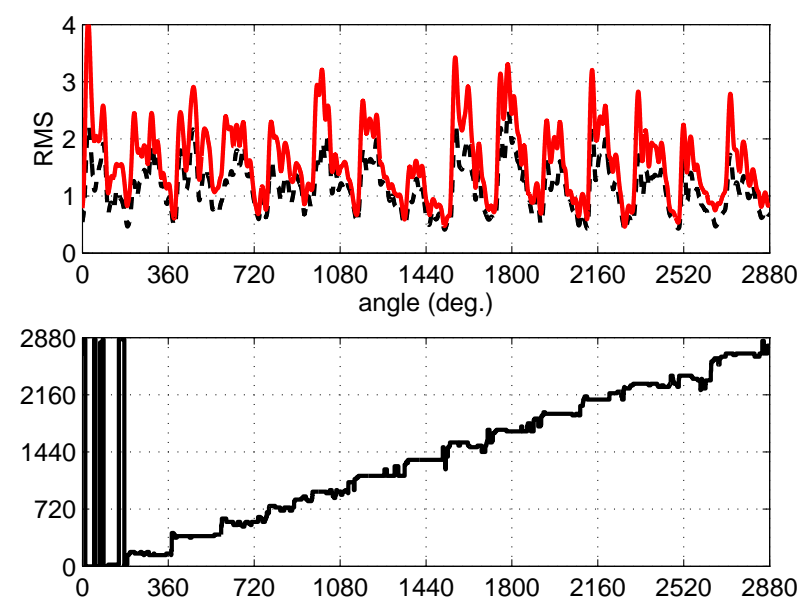

Fig. 8. Gears test rig. Top : RMS value of the ASA(dashed black) and maximized MTSA (solid red) of the microphone signal. Bottom : corresponding synchronization angle maximizing the MTSA.

gear.

This maximization is realized here for the RMS value of the total signal, but it can also be processed for band-pass filtered signals, or even on time frequency maps (tfm), to get more precise or specific results (see figure 9 in the following section):

$$
\theta_{t f m}^{\max }(t, f)=\underset{\theta \in[0 \ldots \Theta]}{\operatorname{argmax}}\left(\operatorname{MTSA}_{t f m}(\theta, t, f)\right),
$$

where $\operatorname{MTSA}_{t f m}(\theta, t, f)$ is the time frequency map (spectrogram for instance) of the MTSA at synchronization angle $\theta$. The maximized time frequency map of the MTSA is thus equal to

$$
\operatorname{MTSA}_{t f m}^{\max }(t, f)=\operatorname{MTSA}_{t f m}\left(\theta_{t f m}^{\max }(t, f), t, f\right)
$$

\subsection{Fuzzy or not fuzzy cyclostationarity?}

The signals can be considered to be fuzzy cyclostationary in the time domain if the moving synchronous average is not constant. Practically, it is the case if the cycle length does not correspond to a constant number of time samples. For signals sampled in angle, the fuzzy characteristic is induced by the timeangle duality of the determinism. It has been previously established [4] that the ADP was inducing a bias on the deterministic part, acting like a damping effect, with a damping ratio increasing with the frequency :

$$
B\left(\omega, t, \sigma_{\nu}\right)=e^{-\omega^{2} t^{2} \sigma_{\nu}^{2}}
$$

This is not exactly the shape of a physical damping, the exponential decreasing being in $e^{-\delta t^{2}}$ and not in $e^{-\delta t}$. However, a comparison can be made between 
the physical damping of the structure and the 'virtual' damping caused by the bias. An energy criterion can be defined to determine if the effect of the bias is significant :

$$
\rho=\frac{\int_{0}^{\infty} e^{-\epsilon \omega t} e^{-\omega^{2} t^{2} \sigma_{\nu}^{2}} d t}{\int_{0}^{\infty} e^{-\epsilon \omega t} d t}
$$

where the numerator corresponds to the integration of the envelope of the ADP of the vibratory structure's response to an event occurring at $t=0$, and the denominator to the integration of the envelope of the TDP synchronized with the considered event. $\epsilon$ stands for the considered structure's damping ratio. If this ratio is significantly lower than one, the bias will be considered as significant. The calculation of (17) leads to

$$
\rho=\kappa e^{\kappa^{2}} \operatorname{erfc}(\kappa) \sqrt{\pi}, \quad \text { with } \kappa=\frac{\epsilon}{\sigma_{\nu} \sqrt{2}}
$$

where $\operatorname{erfc}(\kappa)$ is the complementary error function defined by

$$
\operatorname{erfc}(\kappa)=\frac{2}{\sqrt{\pi}} \int_{\kappa}^{\infty} e^{-x^{2}} d x
$$

It is interesting to note that the value of $\rho$ is determined by the ratio between the physical damping and the normalized standard deviation of the instantaneous speed $\epsilon / \sigma_{\nu}$. Practically, the following numerical observation will be emphasized :

$$
\rho>0.95 \Leftrightarrow \epsilon>4 \sigma_{\nu}
$$

The bias induced by an angular approach will be significant if the damping ratio is four times lower than the speed fluctuation rate. Below this value, the angle sampled vibration signal will be considered as fuzzy cyclostationary.

The result for acoustic signals is not so straightforward from the theoretical point of view, the envelope depending on the damping ratio $\epsilon$, but also on the acoustic radiation operator, and on the reverberation of the room in which signals are recorded. It can be assumed however that acoustic signals are more subject to fuzzy cyclostationarity than vibration signals. This assumption is illustrated here with signals from the diesel engine case. The time frequency map of the ASA and the maximized time frequency map of the MTSA are drawn in figure 9 . these maps are very similar, even if a difference in magnitude is observed above $2 \mathrm{kHz}$, where the maximized MTSA map is more energetic. The time frequency maps are integrated over the whole cycle, in order to assess the energy loss of the ASA as a function of the frequency. The ratio between the energy of the ASA and of the maximized MTSA is drawn in figure 10, it 

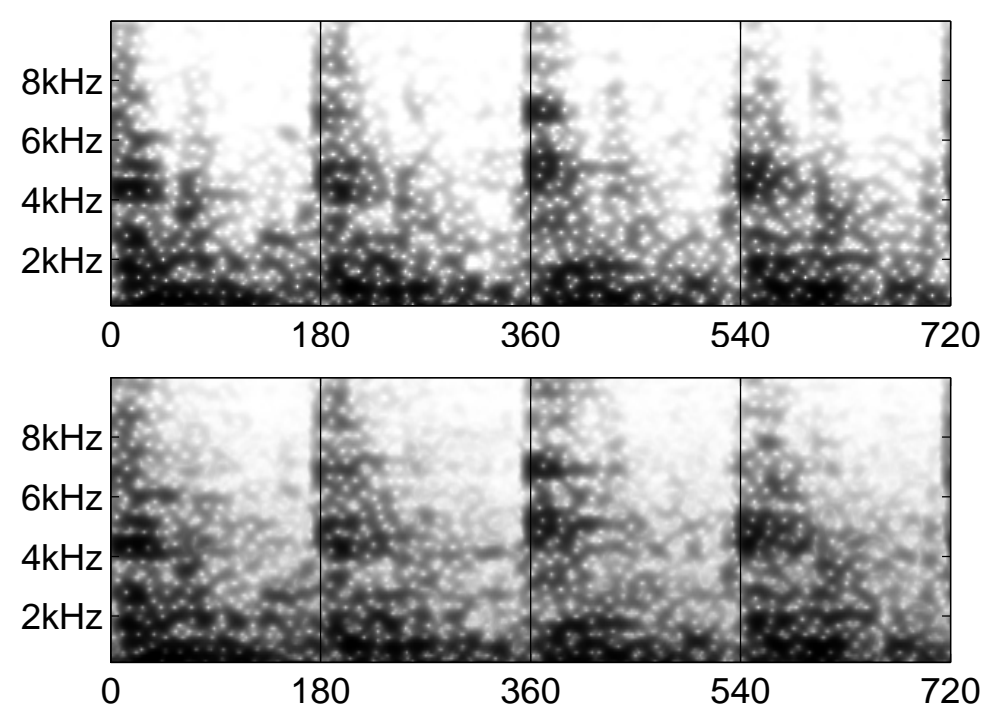

Fig. 9. Time frequency analysis of the synchronous averages of the microphone signal (diesel engine case). Time frequency map of the ASA (top), and maximized time frequency map of the MTSA (bottom). Vertical black lines stand for TDC in the four cylinders.

represents the energy loss of the ASA caused by cycle-to-cycle speed fluctuations for the microphone (gray curve) and for the accelerometer (black curve). Considering the microphone case, this ratio decreases from $0 \mathrm{~dB}$ in low frequency to about $-6 \mathrm{~dB}$ at $10 \mathrm{kHz}$, while remaining between 0 and $-0.5 \mathrm{~dB}$ on the whole frequency range for the accelerometer. It means that speed fluctuations affects significantly the microphone ASA but not the accelerometer ASA.

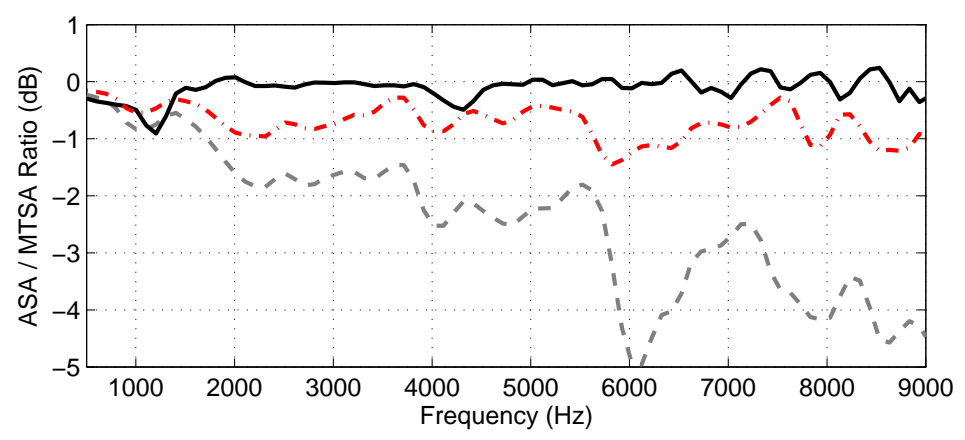

Fig. 10. ASA/MTSA energy ratio for the engine signals. Accelerometer (solid black), microphone (dashed gray), time-shifted microphone (dash-dotted red).

\subsection{Correction of the acoustic propagation delay of microphone signals}

An important part of the energy loss for the microphone ASA can be attributed to the time delay caused by the acoustic propagation from the source of noise to the microphone position. This delay does not affect the TSA or the 
MTSA, because this delay is constant in time, and thus exactly the same from cycle to cycle. For the ASA, this delay is problematic because its duration is modified in angle in case of cycle-to-cycle speed fluctuations, inducing random jitter on the acoustic signal in angle. This issue is discussed in the paper by Stander and Heyns [8] for the case of vibration signals. These authors introduced a method to compensate the phase shift of the vibration signals in angle due to speed fluctuations, based on a phase domain averaging. In the present work, the time delay caused by the acoustic propagation has been considered as an a priori information, based on the knowledge of the source-microphone distance and the celerity of acoustic waves. To compensate for this delay, a simple time shifting operation has been carried out on the microphone signal before the angular resampling :

$$
S(t)=s\left(t+\frac{r}{c}\right)
$$

where $r$ and $c$ are representing respectively the source to microphone distance and the speed of sound. This time-shifting operation is easy to implement if signals are recorded using a time sampling, and re-sampled in angle in postprocess. The operation can be more difficult if signals are directly sampled in angle. Another difficulty is that the source is generally not punctual, the distance $r$ is thus not exactly determined. Considering the acoustic radiation phenomenon, $r$ could be chosen between the minimum and maximum sourcemicrophone distance.

This time-shifting operation has been carried out on the engine signals. The microphone was at about $1 \mathrm{~m}$ from the geometric center of the engine. Signals have been recorded using a time sampling at $102400 \mathrm{~Hz}$, the time shifting operation consisted in removing the 300 first samples of the microphone signal, and the 300 last samples of the angle encoder signal in order to keep equal signal lengths. The energy of the resulting ASA is drawn in figure 10 (dashed red) as a function of the frequency, relatively to the energy of the maximized MTSA. The time-shifting operation has significantly increased the energy of the ASA, of about $2 \mathrm{~dB}$ at $5 \mathrm{kHz}$ and $3 \mathrm{~dB}$ at $9 \mathrm{kHz}$. The ratio between the ASA of the time-shifted signal and the maximized MTSA is less than $1 \mathrm{~dB}$ on the whole frequency range. It remains lower than the ratio obtained for the accelerometer, because of the effects of acoustic radiation and room response.

\section{Conclusion}

Angular sampling is often chosen for the cyclostationary analysis of signals recorded on rotating machines. The major advantage to treat signals sampled in angle is that each cycle realization has exactly the same length, even in case of cycle-to-cycle rotation speed fluctuations. However, using an angular 
sampling can lead to an underestimation of the deterministic response of the structure, whose impulse response is defined in the time domain. In such cases, the time sampling can be an alternative, but the the estimation of the TSA (time synchronous average) depends on the chosen synchronization angle. The computation of the TSA can be realized for various synchronization angles in the cycle, this has been called the moving time synchronous average (MTSA) in this work. The study of this MTSA, taking advantage of cycle-to-cycle instantaneous speed fluctuations, can provide useful information for the analysis and diagnosis of rotating machines. It gives also an estimation of the angular synchronous average energy loss caused by cycle-to-cycle speed fluctuations. It has been shown that this energy loss can be attenuated for acoustic signals by a simple time-shifting operation applied before an angular (re)sampling, to correct the time delay induced by the acoustic propagation.

\section{Acknowledgments}

Authors want to express their gratitude to their colleague François Girardin for the helpful discussions about the revision of this work.

\section{Appendix : Assessment of the Instantaneous Angular Speed of the mean cycle}

The IAS $\Omega(\theta)$ of a rotating machine is generally estimated using an angle encoder, generating a square signal with a period equal to the angular resolution $\Delta \theta$. The IAS is computed using an estimation of the time duration of each period of this signal $\Delta t(\theta)$.

$$
\Omega(\theta)=\frac{\Delta \theta}{\Delta t(\theta)}
$$

Different methods used to assess $\Delta t(\theta)$ are given in [7]. Considering that $\theta=0$ correspond to $t=0$, the elapsed time at angle $\theta$ is equal to

$$
t=f(\theta)=\int_{0}^{\theta} \frac{d \alpha}{\Omega(\alpha)}
$$

Considering a cycle length define in angle by $\Theta$, the time duration of the average cycle is equal to

$$
\bar{T}=\langle f((k+1) \Theta)-f(k \Theta)\rangle_{k}
$$

where $\langle.\rangle_{k}$ stands for the averaging process over cycle realizations. 
The averaged angle to time function is defined, for $0<\theta<\Theta$, as follows :

$$
\begin{aligned}
\bar{f}(\theta) & =\langle f(k \Theta+\theta)-f(k \Theta)\rangle_{k}=\left\langle\int_{k \Theta}^{k \Theta+\theta} \frac{d \alpha}{\Omega(\alpha)}\right\rangle_{k} \\
& =\int_{0}^{\theta}\left\langle\frac{1}{\Omega(\alpha+k \Theta)}\right\rangle_{k} d \alpha=\int_{0}^{\theta} \frac{d \alpha}{\bar{\Omega}(\alpha)},
\end{aligned}
$$

where $\bar{\Omega}$ stands for the IAS of the mean cycle :

$$
\bar{\Omega}(\theta)=\left\langle\Omega(\theta+k \Theta)^{-1}\right\rangle_{k}^{-1} \text { for } 0<\theta<\Theta .
$$

Note that the IAS of the mean cycle is not a cyclic average of the IAS, that is defined by the previous equation without ${ }^{-1}$ superscripts. The interesting property of the IAS of the mean cycle is that its time duration is equal to the average duration of a cycle $(\bar{f}(\Theta)=\bar{T})$, which is not exactly the case for the cyclic average of the IAS. The difference is however negligible for small cycle to cycle speed fluctuations.

Practically, the IAS of the mean cycle is assessed as follows

$$
\bar{\Omega}(\theta)=\frac{\Delta \theta}{\overline{\Delta t}(\theta)}=\frac{\Delta \theta}{\langle\Delta t(\theta+k \Theta)\rangle_{k}} \text { for } 0<\theta<\Theta .
$$

Let now $\epsilon(\theta)$ represent the cycle to cycle relative fluctuation of $\Delta t(\theta)$ :

$$
\Delta t(\theta+k \Theta)=\overline{\Delta t}(\theta)(1+\epsilon(\theta+k \Theta)) \text { for } 0<\theta<\Theta .
$$

The fluctuation of the IAS can be derived as follows, for small relative fluctuations

$$
\Omega(\theta+k \Theta)=\frac{\Delta \theta}{\overline{\Delta t}(\theta)(1+\epsilon(\theta+k \Theta))} \approx \frac{\Delta \theta}{\overline{\Delta t}(\theta)}(1-\epsilon(\theta+k \Theta))=\bar{\Omega}(\theta)(1+\nu(\theta+k \Theta))
$$

Thus, if the cycle to cycle relative fluctuation of the IAS is small, it is equivalent to the cycle to cycle relative fluctuation of $\Delta t(\theta)$ :

$$
\sigma_{\nu}(\theta)=\sigma_{\epsilon}(\theta)=\sqrt{\left\langle\left(\frac{\overline{\Delta t}(\theta)-\Delta t(\theta+k \Theta)}{\overline{\Delta t}(\theta)}\right)^{2}\right\rangle_{k}} \text { for } 0<\theta<\Theta .
$$

\section{References}

[1] S. Braun and B. Seth. Analysis of repetitive mechanism signatures. Journal of Sound and Vibration, 70(4):513-522, 1980. 
[2] J. Antoni. Cyclostationarity by examples. Mechanical Systems and Signal Processing, 23:987-1036, 2009.

[3] S. Braun. The synchronous (time domain) average revisited. Mechanical Systems and Signal Processing, 25(4):1087 - 1102, 2011.

[4] Q. Leclère, L. Pruvost, and E. Parizet. Angular and temporal determinism of rotating machine signals: The diesel engine case. Mechanical Systems and Signal Processing, 24(7):2012 - 2020, 2010. Special Issue: ISMA 2010.

[5] F. Bonnardot. Comparaison entre les analyses angulaire et temporelle des signaux vibratoires de machines tournantes. Etude du concept de cyclostationnarité floue. PhD thesis, Institut National Polytechnique de Grenoble, France, 2004.

[6] L. Pruvost, Q. Leclère, and E. Parizet. Diesel engine combustion and mechanical noise separation using an improved spectrofilter. Mechanical System and Signal Processing, 23:2072-2087, 2009.

[7] K. R. Fyfe and E. D. S. Munck. Analysis of computer order tracking. Mechanical Systems and Signal Processing, 11(2):187-205, 1997.

[8] C.J. Stander and P.S. Heyns. Transmission path phase compensation for gear monitoring under fluctuating load conditions. Mechanical Systems and Signal Processing, 20(7):1511 - 1522, 2006. 